Article

\title{
Exploring Shared Susceptibility between Two Neural Crest Cells Originating Conditions: Neuroblastoma and Congenital Heart Disease
}

\author{
Alessandro Testori ${ }^{1,2}$, Vito A. Lasorsa ${ }^{1,2} \oplus$, Flora Cimmino ${ }^{1,2}$, Sueva Cantalupo ${ }^{3}$, \\ Antonella Cardinale ${ }^{1,2}$, Marianna Avitabile ${ }^{1,2} \mathbb{D}$, Giuseppe Limongelli ${ }^{4}$,

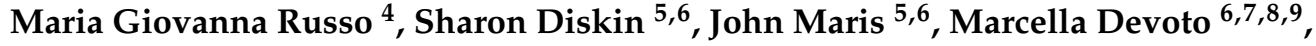 \\ Bernard Keavney ${ }^{10,11}$, Heather J. Cordell ${ }^{12}$, Achille Iolascon ${ }^{1,2}$ (D) and Mario Capasso $1,2,3, *$ (D) \\ 1 Dipartimento di Medicina Molecolare e Biotecnologie Mediche, Università degli Studi di Napoli Federico II, \\ 80131 Naples, Italy \\ 2 CEINGE Biotecnologie Avanzate, 80145 Naples, Italy \\ 3 IRCCS SDN, Istituto di Ricerca Diagnostica e Nucleare, 80143 Naples, Italy \\ 4 Division of Cardiology, Università degli Studi della Campania "Luigi Vanvitelli" - AO dei Colli, Presidio \\ Monaldi, 80121 Naples, Italy \\ 5 Division of Oncology and Center for Childhood Cancer Research, The Children's Hospital of Philadelphia, \\ Philadelphia, PA 19104, USA \\ 6 Department of Pediatrics, The Perelman School of Medicine, University of Pennsylvania, \\ Philadelphia, PA 19104, USA \\ 7 Division of Genetics, The Children's Hospital of Philadelphia, Philadelphia, PA 19104, USA \\ 8 Department of Translational and Precision Medicine, University of Rome "La Sapienza", 00185 Rome, Italy \\ 9 Department of Biostatistics, Epidemiology and Informatics, Perelman School of Medicine, University of \\ Pennsylvania, Philadelphia, PA 19104, USA \\ 10 Division of Cardiovascular Sciences, School of Medical Sciences, Faculty of Biology, Medicine and Health, \\ University of Manchester, Manchester M13 9PL, UK \\ 11 Manchester University NHS Foundation Trust, Manchester Academic Health Science Centre, \\ Manchester M20 4BX, UK \\ 12 Institute of Genetic Medicine, Newcastle University, Central Parkway, Newcastle upon Tyne NE1 3BZ, UK \\ * Correspondence: mario.capasso@unina.it
}

Received: 7 May 2019; Accepted: 26 August 2019; Published: 30 August 2019

check for updates

\begin{abstract}
In the past years, genome wide association studies (GWAS) have provided evidence that inter-individual susceptibility to diverse pathological conditions can reveal a common genetic architecture. Through the analysis of congenital heart disease (CHD) and neuroblastoma (NB) GWAS data, we aimed to dissect the genetic susceptibility shared between these conditions, which are known to arise from neural crest cell (NCC) migration or development abnormalities, via identification and functional characterization of common regions of association. Two loci (2q35 and 3q25.32) harbor single nucleotide polymorphisms (SNPs) that are associated at a $p$-value $<10^{-3}$ with conotruncal malformations and ventricular septal defect respectively, as well as with NB. In addition, the lead SNP in 4p16.2 for atrial septal defect and the lead SNP in 3q25.32 for tetralogy of Fallot are less than $250 \mathrm{~Kb}$ distant from the lead SNPs for NB at the same genomic regions. Some of these shared susceptibility loci regulate the expression of relevant genes involved in NCC formation and developmental processes (such as BARD1, MSX1, and SHOX2) and are enriched in several epigenetic markers from NB and fetal heart cell lines. Although the clinical correlation between NB and CHD is unclear, our exploration of a possible common genetic basis between NB and a subset of cardiac malformations can help shed light on their shared embryological origin and pathogenetic mechanisms.
\end{abstract}

Keywords: genome wide association studies; neuroblastoma; congenital heart disease 


\section{Introduction}

Neuroblastoma (NB) is an embryonic tumor arising from the sympathetic nervous tissue and is among the most frequent cancers diagnosed in early infants, accounting for $13 \%$ of all deaths due to childhood malignancies [1]. Its etiology is due to an overgrowth in the sympathetic ganglion where neural crest derived progenitors reside. Whereas familial NB is rare [2], sporadic NB has a higher incidence: The study of its genetic susceptibility can therefore benefit from a more abundant cohort of patients and has thus been largely investigated by means of genome wide association studies (GWAS) [3,4] and candidate gene approaches [5-7].

Congenital heart disease (CHD) is one of the most frequent inborn disorders in infants, affecting 7 in 1000 live births and is a major cause of childhood death and long term morbidity [8]. Complex genetic mechanisms underlie cardiac development and its anomalies, and a number of different defects could be the cause-such as migration defects, reduced specification or overproduction of neural crest-derived mesenchymal cell types-and efforts have been made to try and elucidate causative variants affecting these conditions [9-12].

Neural crest cells (NCC) development and migration abnormalities have been conjectured to be implicated in the genesis of both CHD and NB [13-16], and there are case reports in the literature of patients affected with both of these conditions simultaneously [17]. George and colleagues [18] demonstrated that children affected with NB have a higher prevalence of CHD; however, van Engelen and colleagues [19] have denied evidence of association between these two conditions. A review of more than 1900 cases showed that NBs account for approximately $17 \%$ of the malignancies seen in Costello and Noonan syndromes [20], a disorder characterized by diverse tissue and organ defects, including CHD [21]. Lombardo and colleagues [22] very recently reported an association between CHD and mutations in PHOX2B, a susceptibility gene for familial NB [23]. In spite of some negative evidence, it is possible that NB and CHD share susceptibility loci but that their phenotypes are not highly penetrant in individuals with certain susceptible mutations.

Demonstrating a correlation between NB and CHD could provide useful information to patients suffering from these conditions, including the opportunity of specific genetic counseling addressing the possible onset of the other disease. Although epidemiological studies are a powerful tool for addressing this question, genome wide association studies (GWAS) can provide a deeper level of understanding of the genetics underlying phenotypic traits, including pathological conditions. The accumulation of large-scale genomic datasets has led to the detection of novel loci associated with diverse traits and enhanced the study of shared genetic factors across phenotypes, but a thorough characterization of these identified loci would be advisable, both at the genetic and epigenetic level. Using data from different conditions can reveal the presence of common genetic risk factors and shared causal pathways, thus improving our understanding of disease.

Given NB and CHD common embryological derivation from NCC [21,24], we analyzed GWAS results for these traits in order to evaluate the extent of shared genetics between NB and seven $\mathrm{CHD}$ conditions: atrial septal defect/patent foramen ovale (ASD/PFO), conotruncal malformations (CM), double outlet right ventricle (DORV), left-sided malformations (LH), transposition of the great arteries (TGA), tetralogy of Fallot (ToF), and ventricular septal defect (VSD).

\section{Materials and Methods}

\subsection{Neuroblastoma GWAS Summary Statistics}

GWAS summary statistics were taken from the work of McDaniel and colleagues [25]. These refer to a European-American cohort of 2101 cases and 4202 matched controls (Table 1) assayed with Illumina HumanHap550 v1, HumanHap550 v3, and Human610 single nucleotide polymorphism (SNP) arrays. Genotype phasing was performed using SHAPEIT v2.r790 [26] and data from 1000 Genomes Phase 1 Release 3. Subsequent imputation was performed genome-wide using IMPUTE2 v2.3.1 [27] for all SNPs and indel variants annotated in 1000 Genomes Phase 1 Release 3. Only SNPs with minor allele 
frequency (MAF) $>0.01$ and info score $>0.8$ were considered. Manhattan plot of the NB GWAS is available in Figure S1 and characteristics of patients are summarized in Table S1.

Table 1. Data sets used in this study.

\begin{tabular}{ccc}
\hline Condition & Cases & Controls \\
\hline ASD/PFO & 340 & 5159 \\
CM & 151 & 5159 \\
DORV & 96 & 5159 \\
LH & 387 & 5159 \\
NB & 2101 & 4202 \\
TGA & 207 & 5159 \\
ToF & 835 & 5159 \\
VSD & 191 & 5159
\end{tabular}

Number of cases and controls for each dataset used. ASD/PFO: atrial septal defect/patent foramen ovale; CM: conotruncal malformations; DORV: double outlet right ventricle; LH: left-sided malformations; NB: neuroblastoma; TGA: transposition of the great arteries; ToF: tetralogy of Fallot; VSD: ventricular septal defect.

\subsection{CHD Genotypes}

Genotypes from 5159 controls and patients with seven different subtypes of CHD, namely atrial septal defect/patent foramen ovale (ASD/PFO, 340 cases), conotruncal malformations (CM, 151 cases), double outlet right ventricle (DORV, 96 cases), left-sided malformations (LH, 387 cases), transposition of the great arteries (TGA, 207 cases), tetralogy of Fallot (ToF, 835 cases), and ventricular septal defect (VSD, 191 cases), were those included in the work of Cordell and colleagues [9]. Manhattan plots of the CHD GWAS are available in Figure S1.

\subsection{CHD Genotypes Imputation}

We used the Michigan Imputation Server [28] to perform imputation on the CHD datasets (reference panel: 1000G Phase 3 v5).

\subsection{CHD Association Analysis}

Dosage vcf files from the imputation output were fed to SNPTEST v2.5.4 beta1 [29] software and frequentist association test was used to compute summary statistics.

\subsection{Evaluation of the Extent of Shared Genetics}

The first step of our workflow (Figure 1) was the evaluation of the genome-wide shared genetics between NB and CHD. To this end, we pruned the whole set of common SNPs based on linkage disequilibrium (LD) with plink v1.9 [30] using a $\mathrm{r}^{2}$ threshold of 0.2 (plink -indep-pairwise function; parameters used are: $\mathrm{r}^{2}=0.2$; window size $=500 \mathrm{~Kb}$; step size $=20$ ) in order to consider only SNPs in approximate linkage equilibrium and evaluated the number of independent SNPs with association $p$-values above and below several thresholds $(0.05,0.01,0.005,0.001,0.0005,0.0001)$ in NB and in each CHD condition. This procedure prevents the overestimation of association signals due to LD structure (such as when multiple signals are present from high LD regions). If the two conditions do not share a genetic basis, these values should not deviate from random expectation. A $2 \times 2$ table for each $p$-value cutoff was created and one-sided Fisher exact tests were used as the statistical measure of significance and strength of association. We also ran simulations to assess the validity of our results: For each CHD condition, each SNP was randomly assigned a $p$-value from the list of observed $p$-values deriving from the association analysis for that condition. We repeated this a thousand times, and an empirical $p$-value was calculated from the proportion of simulations having a number of SNPs below a given $p$-value threshold in both datasets (NB and one CHD type) greater than or equal to the observed number of SNPs below the same $p$-value threshold in both real datasets (NB and one CHD type) under consideration. 


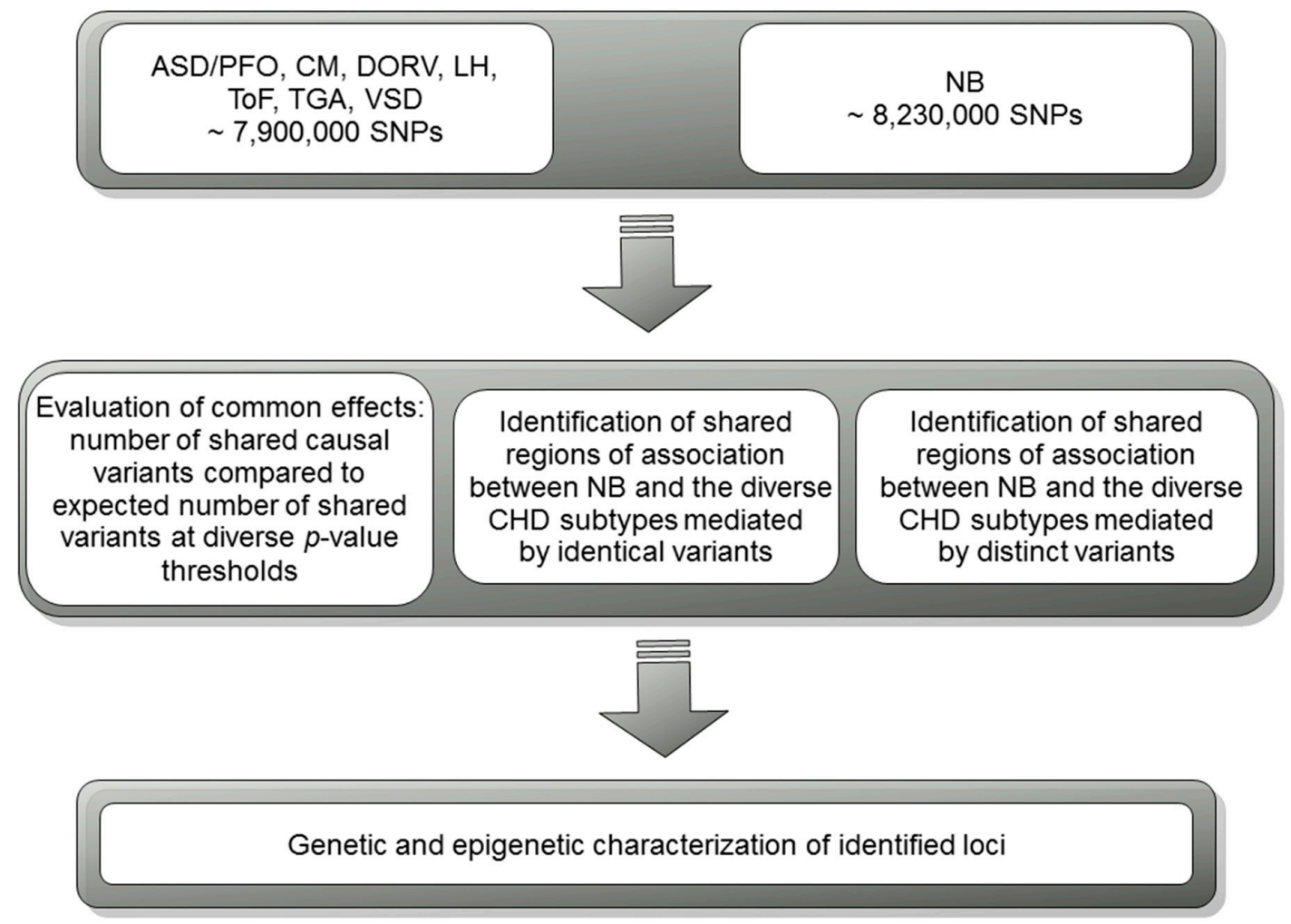

Figure 1. Study design and workflow.

\subsection{Identification of Colocalizing Association Signals}

To identify shared association signals between NB and CHD, SNPs with an association $p$-value $<10^{-3}$ [31] in both NB and at least one CHD dataset were selected, and shared association signals were defined as those regions containing at least 10 such SNPs within a distance of less than $100 \mathrm{~Kb}$.

To identify possibly colocalizing but distinct association signals, we selected SNPs with an association $p$-value $<10^{-5}$ in NB or at least one CHD dataset, and candidate regions were identified if at least 10 SNPs within a distance of less than $100 \mathrm{~Kb}$ were present. The threshold of $p$-value $<10^{-5}$ was chosen as it corresponds to an average of 1 false-positive association per GWAS in European populations [32]. The distance between lead SNPs of these candidate regions in NB and in the CHD conditions was then used to evaluate these potential colocalization signals deriving from distinct variants.

We also ran simulations to assess the significance of these distinct, colocalizing signals by randomly reshuffling the location of the associated regions in our NB dataset while keeping their size fixed: An empirical $p$-value was calculated from the proportion of simulations having a number of regions less than $250 \mathrm{~Kb}$ distant between NB and CHD greater than or equal to the real datasets.

A method proposed by Pickrell and colleagues [33] was also used to detect colocalizing regions. The algorithm generates posterior probabilities through a Bayesian approach for the hypotheses that the region harbors one variant associated to both, to only one or to none of the traits, or that the region contains one variant associated only to one trait and another variant associated only to the other trait.

\subsection{Enrichment of Epigenetic Signatures in Susceptibility Loci}

Enrichment in epigenetic features of several cell types related to neural crest cells (NCC) and heart, and NCC derived tumors (NB and melanoma) was computed through R VSE package from CRAN [34]. Briefly, it creates a network from SNPs that accounts for LD structure and generates a null model by sampling SNPs from a comprehensive pool of tag SNPs, thus recreating the same LD clusters as in the real data, matching each associated variant set to a random variant set with the same characteristics. Haploreg [35] was also used to perform enhancer enrichment analysis on sets of variants (binomial 
test), using as background frequency the overlap from 1000 Genome variants with a frequency above $5 \%$ in any population.

\section{8. eQTL Analysis}

LinDA [36] was used to identify genes regulated by variants of interest, as well as tissues involved. This tool takes as input a list of variants and queries 199 datasets belonging to 53 projects, comprising 15 human populations and 33 body districts, resulting in 486,244 eQTLs and 36,768 eGenes.

\section{Results}

\subsection{Evaluation of the Genome Wide Extent of Shared Genetic Association}

To evaluate the genome-wide shared genetic signals between NB and CHD, we selected a subset of independent SNPs in approximate linkage equilibrium with each other and evaluated for each condition the number of SNPs with association $p$-value above and below different thresholds. We used Fisher exact test and simulation analysis to evaluate whether NB and each CHD condition in turn share more SNPs above and below the $p$-value thresholds than expected by chance [37] (see Materials and Methods Section 2.5). We found some evidence of shared association signals between NB and ASD/PFO, between NB and CM, and between NB and VSD (Table 2, Figure 2, Tables S2 and S3). As reported in Table 2, SNPs with $p$-value less than 0.01 are shared more frequently than expected between NB and all CHD datasets (Fisher exact test $p$-value $=0.02$ ). Common association signals are also observed for low $p$-value thresholds when considering NB and CM $(<0.005$; Fisher exact $p$-value $=0.04)$ and when considering NB and VSD $(<0.0005$; Fisher exact $p$-value $=0.05)$ and for high $p$-value thresholds when considering NB and ASD/PFO $(<0.05$; Fisher exact $p$-value $=0.02)$.

Table 2. Evaluation of the extent of shared genetic effects between neuroblastoma (NB) and congenital heart disease (CHD).

\begin{tabular}{cccc}
\hline Dataset & $p$-value Threshold & Fisher Test $p$-value & Odds Ratio \\
\hline ALL & 0.0001 & 1 & 0 \\
ALL & 0.0005 & 1 & 0 \\
ALL & 0.001 & 1 & 0 \\
ALL & 0.005 & 0.92 & 0.46 \\
ALL & 0.01 & 0.02 & 1.56 \\
ALL & 0.05 & 0.84 & 0.94 \\
ASD/PFO & 0.0001 & 1 & 0 \\
ASD/PFO & 0.0005 & 1 & 0 \\
ASD/PFO & 0.001 & 1 & 0 \\
ASD/PFO & 0.005 & 0.64 & 0.89 \\
ASD/PFO & 0.01 & 0.52 & 1.01 \\
ASD/PFO & 0.05 & 0.02 & 1.12 \\
CM & 0.0001 & 1 & 0 \\
CM & 0.0005 & 1 & 0 \\
CM & 0.001 & 0.18 & 4.97 \\
CM & 0.005 & 0.04 & 2.05 \\
CM & 0.01 & 0.23 & 1.23 \\
CM & 0.05 & 0.99 & 0.84 \\
VSD & 0.0001 & 1 & 0 \\
VSD & 0.0005 & 0.05 & 19.45 \\
VSD & 0.001 & 0.15 & 5.86 \\
VSD & 0.005 & 0.27 & 1.43 \\
VSD & 0.01 & 0.53 & 1 \\
VSD & 0.05 & 0.8 & 0.94 \\
\hline
\end{tabular}

The union of all CHD datasets is considered as well as the most significant subtypes from this analysis. After extracting SNPs in approximate linkage equilibrium $\left(\mathrm{r}^{2}<0.2\right)$ from the full set of all common SNPs (see Materials and Methods Section 2.5 for details), for $p$-values ranging from 0.0001 to 0.05 , Fisher exact test was performed for the SNPs above and below $p$-value threshold in NB and in the given condition. 

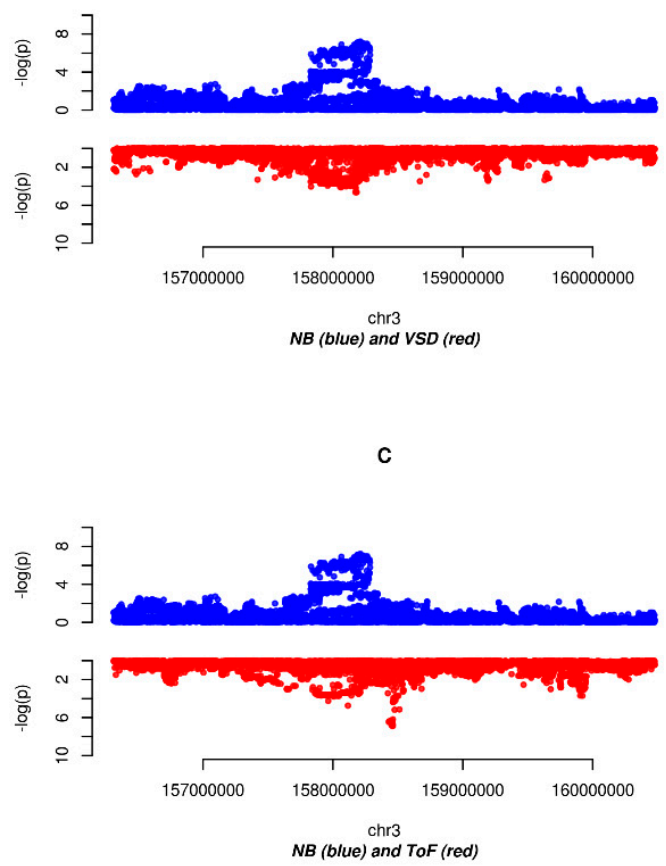

E

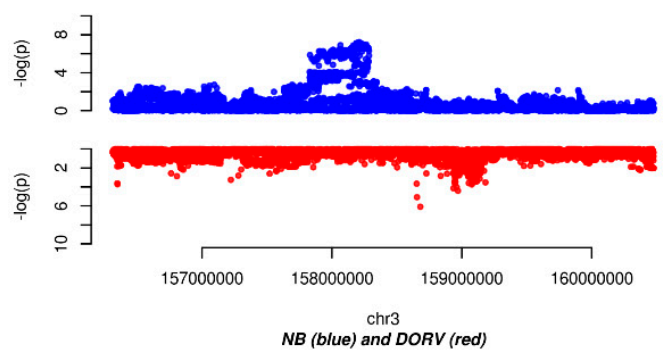

B
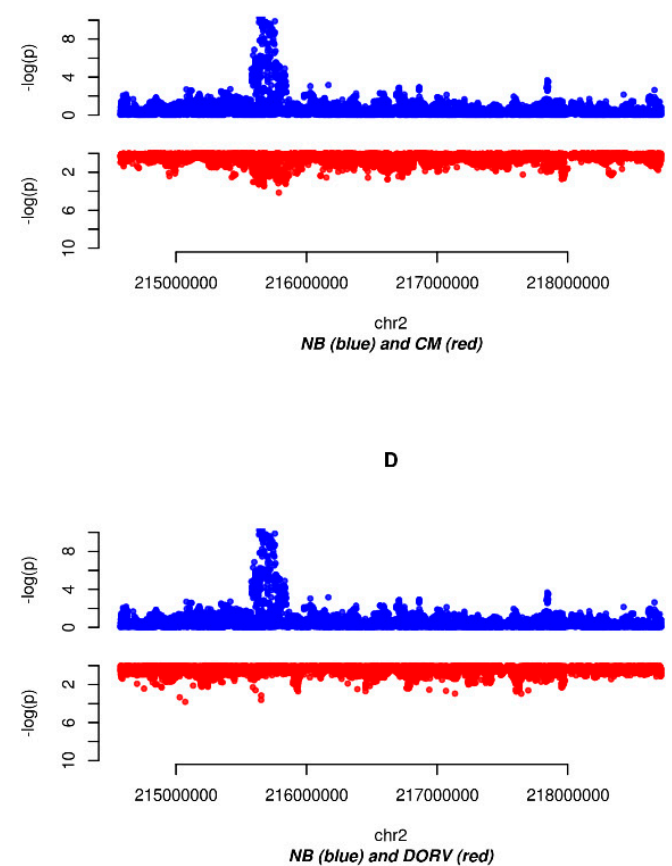

$\mathbf{F}$

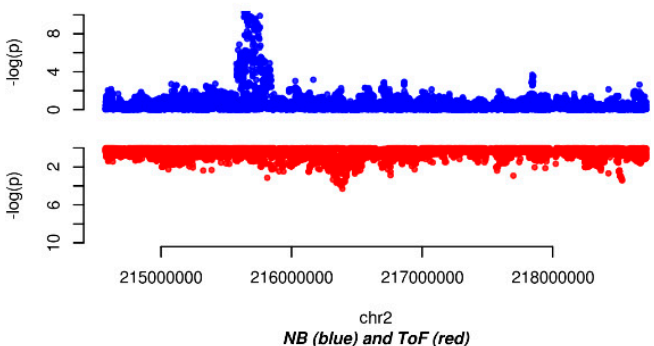

Figure 2. Regional association plots of significant loci described in text. In blue is NB, in red are different subtypes of CHD. (A) NB and VSD at 3q25.32, (B) NB and CM at 2q35, (C) NB and ToF at 3q25.32, (D) NB and DORV at 2q35, (E) NB and DORV at 3q25.32, (F) NB and ToF at 2q35.

\subsection{Identification of Colocalizing Association Signals between NB and CHD}

We defined shared association regions as genomic locations harboring at least 10 SNPs with association $p$-value $<10^{-3}$ in NB and in at least one CHD subtype (see Materials and Methods Section 2.6). With this procedure, we identified two main regions spanning over several $\mathrm{Kb}$ : one shared between NB and VSD (3q25.32; 399 SNPs, Figure 2A) and another one shared between NB and $\mathrm{CM}$ (2q35; 28 SNPs, Figure 2B). Two smaller regions were also identified: 12q21.31 has overlapping association signals in VSD, ASD/PFO, and NB and 14q24.3 has few SNPs which are significant both in NB and in ToF (Table 3). In this last case the direction of effect of the colocalizing SNPs in both datasets is the same, supporting a genuine shared allelic risk; whereas in the other cases the direction of effect is opposite, implying a shared genetic basis [38].

Following recent works that have pointed out the importance of effects mediated by distinct genetic determinants located in the same genomic regions for informing the causal relationship between different traits [33,39-41], we also evaluated evidence of this kind of spurious colocalization between NB and each CHD subtype. On the basis of the physical distance of lead SNPs in significant loci of association in NB and CHD we identified two colocalizing susceptibility regions: one region 
encompassing band 3q25, colocalizing between NB and ToF (Figure 2C and Table 4), and one further region in band 4p16.2 in NB and ASD/PFO (Figure S2 and Table 4, empirical $p$-values $<0.04$ and $<0.03$, respectively). Table $S 4$ reports all regions in the analyzed datasets with $p$-value $<10^{-5}$ and their relative distance.

We also used a Bayesian method designed to test whether some genomic regions may harbor distinct variants associated to multiple traits [33] (see Materials and Methods Section 2.6). We found ten instances with a posterior probability $>0.9$ of containing distinct variants associated to NB and one or more CHD subsets (Table 5). Interestingly some of these identified regions were also identified through the other approaches although in different CHD subtypes: 2q35 has overlapping association signals between NB and CM (Figure 2B) and shows evidence of colocalization of NB with DORV and ToF (Figure 2D,F) and 3q25.32 has overlapping association signals between NB and VSD (Figure 2A) and shows evidence of colocalization of NB with DORV and ToF (Figure 2C,E). Region 4p16 contains both a signal of colocalization between NB and ASD/PFO (see above), as well as a signal of colocalization between NB and DORV. Two further colocalizing regions were identified: 6p22 (colocalizing NB with CM and NB with DORV) and 11p15 (colocalizing NB with DORV and NB with ToF) (Table 5 and Figure S2).

\subsection{Enrichment in Epigenetic Markers in Colocalizing Regions}

Epigenetic features overlapping genetic polymorphisms can help predict in which cell tissue that variant is likely to act [42]. Therefore we evaluated enrichment of several epigenetic markers from cell lines and tissues related to neural crest cells, NB, and heart development (see Table S5 for the complete list) in the set of the most significant SNPs previously identified (i.e., SNPs with $p$-value $<10^{-3}$ in NB and in at least one CHD subset in the regions reported in Table 3). In order to account for LD structure and prevent enrichment inflation in case of SNPs residing in high LD blocks, we used the Variant Set Enrichment (VSE) package from CRAN [34]. Results are shown in Figure 3. It can be seen that few NB cell lines (NB69, LAN1, BE2C) are significantly enriched in these regions. Interestingly (Table S6), it can be inferred that $2 \mathrm{q} 35$ is an epigenetic hotspot and has signatures from many cell lines whereas $3 \mathrm{q} 25.32$ has several epigenetic signatures from adrenal and fetal heart, which are also abundant in 2q35. The core 15-state model source for epigenomes in HaploReg [35] also gives evidence of enrichment in fetal heart signatures $(p$-value $=0.024$ ) in these cross-associated variants.

\subsection{Annotation of Colocalizing Regions}

We used the genetic variants from the shared (association $p$-value $<10^{-3}$ ) and distinct (association $p$-value $<10^{-5}$ ) colocalizing signals and queried them for eQTL annotation through LinDA (http: //linda.irgb.cnr.it/). Genes, variants, and tissues are listed in Table 6 and Table S7. This procedure allows to annotate genes from multiple catalogs that are likely regulated by the list of variants given as input in a tissue specific manner. 
Table 3. Shared association regions between neuroblastoma and the diverse CHD subtypes.

\begin{tabular}{|c|c|c|c|c|c|c|c|c|}
\hline Disease & Band & pos hg19 & $\begin{array}{c}\text { SNPs with } \\
p \text {-value }<10^{-3}\end{array}$ & Direction of Effect & Lead SNP NB & $p$-value & Lead SNP CHD Subtype & $p$-value \\
\hline $\mathrm{CM}$ & $2 q 35$ & $215590505-215840829$ & 28 & opposite & rs3768708 & $1.09 \times 10^{-10}$ & rs34206771 & $7.15 \times 10^{-5}$ \\
\hline ASD/PFO & $12 \mathrm{q} 21.31$ & $85606538-85723868$ & 16 & opposite & rs7295242 & $2.75 \times 10^{-4}$ & rs13377665 & $3.71 \times 10^{-4}$ \\
\hline VSD & $12 \mathrm{q} 21.31$ & $85604092-85723868$ & 18 & opposite & rs11116772 & $2.41 \times 10^{-4}$ & rs7954427 & $5.03 \times 10^{-4}$ \\
\hline VSD & $3 q 25.32$ & $157828781-158245883$ & 399 & opposite & rs1978779 & $6.09 \times 10^{-8}$ & rs6441201 & $2.39 \times 10^{-5}$ \\
\hline ToF & $14 \mathrm{q} 24.3$ & 79029133-79059667 & 14 & same & rs4643247 & $5.88 \times 10^{-5}$ & rs7159049 & $7.75 \times 10^{-5}$ \\
\hline
\end{tabular}

For each region is reported the number of SNPs that have an association $p$-value below $10^{-3}$ in both datasets in that genomic region and the direction of effect, the genomic band, the left and right margins of this region, and its range in bases, and the lead SNPs in NB and in the CHD subtype with association $p$-values.

Table 4. Physical distance between lead SNPs in NB and the diverse CHD subtypes.

\begin{tabular}{cccccccccccc}
\hline Band & Disease & Lead SNP & pos hg19 & $\boldsymbol{p}$-value & Disease & Lead SNP & pos hg19 & $p$-value & Distance & $\mathbf{D}^{\prime}$ & $\mathbf{R}^{2}$ \\
\hline $3 \mathrm{q} 25.32$ & NB & rs1978779 & 158211291 & $6.09 \times 10^{-8}$ & ToF & rs75107964 & 158458751 & $1.30 \times 10^{-7}$ & 247,460 & 0.7 & 0.1 \\
4p16.2 & NB & rs11944652 & 4892294 & $1.61 \times 10^{-6}$ & ASD/PFO & rs4689909 & 4643276 & $7.75 \times 10^{-7}$ & 249,018 & 0.1 & 0.01 \\
\hline
\end{tabular}

The table shows only cases in which a lead SNP in a susceptibility locus of NB is less than 250,000 bp away from a lead SNP in a susceptibility locus of at least one CHD subtype.

Table 5. Regions of spurious colocalization between NB and diverse CHD subtypes.

\begin{tabular}{|c|c|c|c|c|c|c|c|}
\hline Disease & Band & pos hg19 & Lead SNP NB & $p$-value NB & Lead SNP CHD & $p$-value CHD & $\mathbf{P P}$ \\
\hline $\mathrm{CM}$ & $6 \mathrm{p} 22.3$ & $21685357-22748186$ & rs4712656 & $6.33 \times 10^{-16}$ & rs147429944 & $7.39 \times 10^{-9}$ & 0.932813 \\
\hline DORV & 11p15.4 & $7436942-8331494$ & rs204926 & $6.91 \times 10^{-12}$ & rs12807437 & $1.71 \times 10^{-3}$ & 0.906466 \\
\hline DORV & $2 q 35$ & 215573795-217714948 & rs2070096 & $3.39 \times 10^{-11}$ & rs116515369 & $2.43 \times 10^{-4}$ & 0.91838 \\
\hline DORV & $3 q 25.33$ & $157312429-159477493$ & rs1978779 & $6.09 \times 10^{-8}$ & 158680170 & $8.16 \times 10^{-7}$ & 0.923649 \\
\hline DORV & $4 \mathrm{p} 16.1$ & $8154534-8733618$ & rs3796727 & $3.19 \times 10^{-9}$ & chr4:8379187:I & $5.06 \times 10^{-3}$ & 0.91279 \\
\hline DORV & $6 \mathrm{p} 22.3$ & 21685357-22748186 & rs4712656 & $6.33 \times 10^{-16}$ & rs115828798 & $1.37 \times 10^{-4}$ & 0.926876 \\
\hline DORV & $6 \mathrm{q} 21$ & $103983460-106054975$ & rs4945714 & $1.28 \times 10^{-8}$ & rs78448955 & $1.44 \times 10^{-3}$ & 0.906372 \\
\hline $\mathrm{ToF}$ & 11p15.4 & $7436942-8331494$ & rs204926 & $6.91 \times 10^{-12}$ & rs6578887 & $3.80 \times 10^{-5}$ & 0.917802 \\
\hline $\mathrm{ToF}$ & $2 q 35$ & 215573795-217714948 & rs2070096 & $3.39 \times 10^{-11}$ & rs13023347 & $5.08 \times 10^{-5}$ & 0.919856 \\
\hline $\mathrm{ToF}$ & $3 q 25.33$ & $157312429-159477493$ & rs1978779 & $6.09 \times 10^{-8}$ & rs75107964 & $1.30 \times 10^{-7}$ & 0.99738 \\
\hline
\end{tabular}

Regions in the table show evidence of association via two distinct variants in NB and in one CHD subtype. 


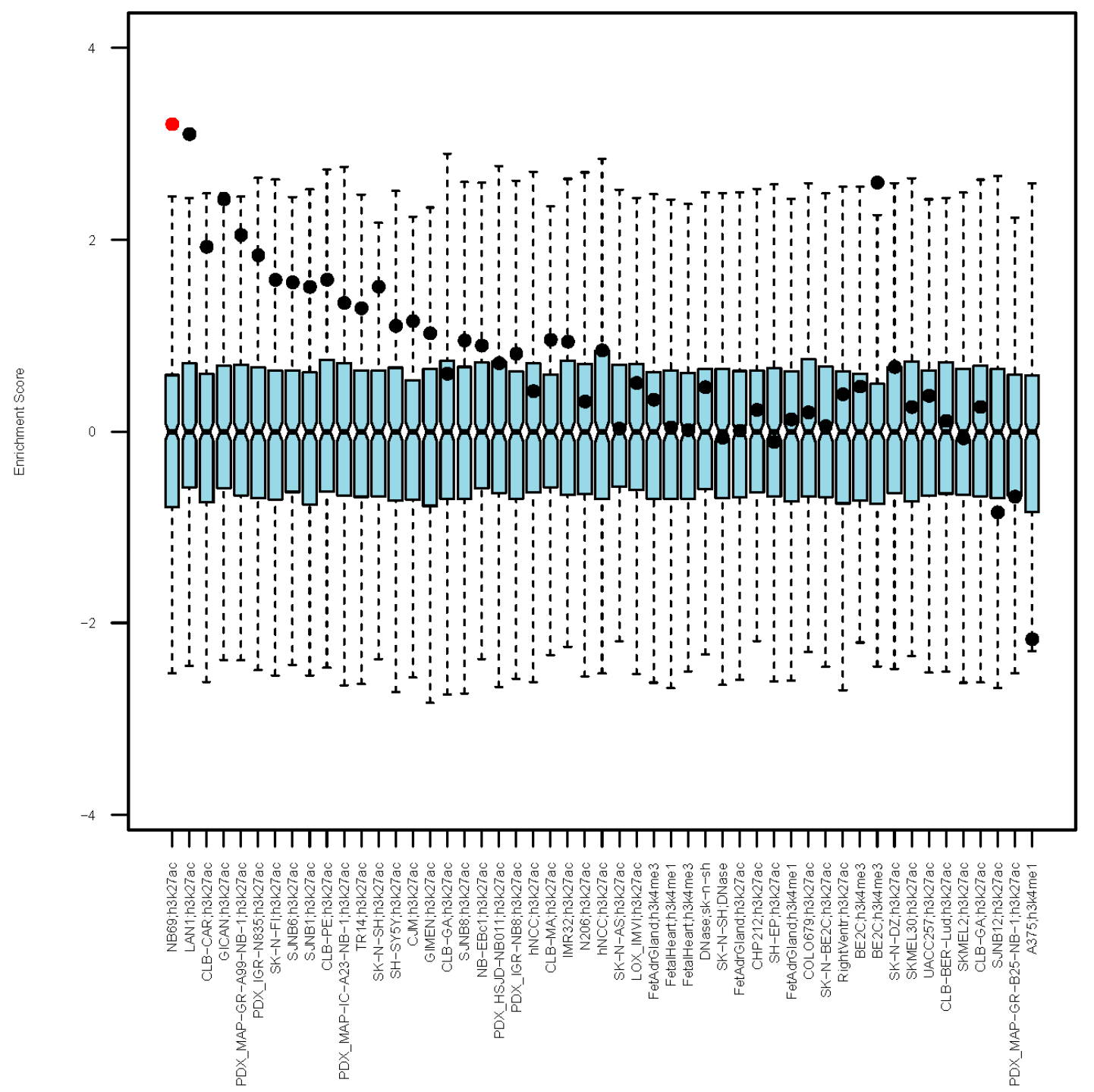

Figure 3. Box plots represent the distribution of overlap of the epigenetic feature under consideration with random sets of markers matched to the real set in terms of numerosity and LD structure. The bar inside each box corresponds to the median enrichment score of the null set. Whiskers span from minimum value to first quartile and from third quartile to maximum value. Dots represent the estimated enrichment in the real set of SNPs considered. One feature still significant after stringent multiple testing correction (Bonferroni corrected $p$-value $<0.01$ ) is marked in red.

Table 6. eQTL mapping performed in shared and colocalizing susceptibility loci.

\begin{tabular}{ccc}
\hline Gene & Band & Number of SNPs \\
\hline BARD1 & 2:q35 & 20 \\
MFSD1 & 3:q25.32 & 47 \\
RARRES1 & $3: \mathrm{q} 25.32$ & 49 \\
RP11-379F4.4 & $3: \mathrm{q} 25.32$ & 47 \\
RP11-538P18.2 & $3: \mathrm{q} 25.32$ & 12 \\
RSRC1 & $3: \mathrm{q} 25.32$ & 159 \\
SHOX2 & $3: \mathrm{q} 25.32$ & 2 \\
HS.276795 & $4: \mathrm{p} 16.2$ & 4 \\
MSX1 & $4: \mathrm{p} 16.2$ & 1 \\
\hline
\end{tabular}

Genes whose expression is affected by SNPs in identified susceptibility loci common to NB and CHD (see Results Section 3.4) are shown. Genomic bands and the number of variants analyzed affecting the expression of these genes is also reported. 


\section{Discussion}

The evaluation of shared association between epidemiologically linked conditions represents a powerful tool for the dissection of common and unique mechanisms in the development of phenotypic traits and the onset of pathological conditions $[43,44]$. On the basis of possible co-occurrence of NB and CHD [18,45] and their common derivation from NCC [21,24], we conducted a co-association study on these conditions, starting from a general evaluation of an excess of shared association signals, to a more detailed analysis of colocalizing association signals. We observed the strongest evidence of shared genetic architecture between NB and VSD, both at a genome-wide level (Table 2 and Figure 2) and at single loci (Tables 3-5), where in band 3q25.32 a region of nearly half Mb harbors 399 SNPs with association $p$-value below $10^{-3}$ in both conditions, which supports a genuine shared effect. This same region also shows evidence of shared association between NB and ToF and between NB and DORV.

Most of the SNPs that we identified in these loci of shared association show an opposite allelic effect. It was reported in the literature that for several conditions with a common pathological basis, shared genomic loci of association (such as the ones resulting from phenotype cross-trait analysis) show an opposite effect in several cases, possibly implying opposite functional changes in different cells/tissues affecting the same molecular trait or pathway [39].

Some of the regions detected by our colocalization analysis include intriguing candidate genes for NB and CHD. MSX1 (4p16.2) is a homeobox gene involved in neural crest specification [46] that has been already identified as a CHD susceptibility gene [47]. Our results suggest that common variants can affect MSX1 expression and can also predispose to NB. The role of MSX1 in NB biology is also supported by a recent paper that demonstrates a signaling axis leading from $P H O X 2 B$ via MSX1 to Delta-Notch and proneural gene expression in NB pathogenesis [48]. Recently, NB has been diagnosed in a child with Wolf-Hirschhorn syndrome, a congenital disorder with characteristic facial features caused by microdeletion of the short arm of chromosome 4 encoding the MSX1 gene [49].

Another relevant gene is SHOX2 (3q25.32), a member of the homeobox family which is one of the major genes involved in the development of the sinoatrial node [50]; its proper function is of crucial relevance for the origin of arrhythmogenic heart disease [51]. Moreover, SHOX2 is implicated in specifying neural systems involved in processing somatosensory information, as well as in face and body structure formation [52,53] and has been reported as involved in Cornelia de Lange syndrome-a condition that implies heart defects [52,54]. The relevance of this gene is supported from its association with eQTLs.

Our results and those from the literature show that the aforementioned genes are involved in developmental processes and that their abnormal functioning due to genetic alterations could predispose to the development of NB and CHD.

eQTL analysis points out the relevance of loci associated at 3q25.32; in fact 3 genes (MLF1, RP11-538P18.2, and RSRC1) are associated with variants relevant in at least four conditions: NB, DORV, ToF, and VSD. MLF1 in particular has been recently described in NB [25] and seems to play an important role in tumorigenesis. MLF1 is highly expressed in heart and has been identified as a novel modulator of cardiomyocyte proliferation [55]. Interestingly, our eQTL analysis using data from left ventricle tissues demonstrates that predisposing NB and CHD variants can affect MLF1 expression.

We found that the known NB susceptibility gene BARD1 (2q35) [4,56] lies in close proximity to a candidate susceptibility locus for CM; copy number alterations at the BARD1 locus have been previously associated to developmental delay, coarctation of aorta and ToF [57], suggesting a role of BARD1 in early organogenesis and heart formation.

The identification of regions of shared susceptibility can help in assigning a hierarchy in the pathogenic mechanisms of related conditions, and functional and epigenetic characterization of common associated SNPs from different traits can contribute to single out loci belonging to shared and unique pathways. Our results suggest a possible common genetic basis between these two NCC originating conditions. However, larger sample sizes and further studies will be needed to validate our results and better elucidate the shared genetic risk factors between NB and CHD. 
Supplementary Materials: The following are available online at http://www.mdpi.com/2073-4425/10/9/663/s1, Figure S1: Manhattan plots of neuroblastoma (NB) and different types of congenital heart disease (CHD), Figure S2: Regional association plots of significant loci described in text, Table S1: Characteristics of patients in the neuroblastoma (NB) cohort, Table S2: Evaluation of the extent of shared genetic effects between NB and congenital heart disease (CHD), Table S3: Evaluation of the extent of shared genetic effects between NB and CHD via simulations, Table S4: Physical distance between lead SNPs in NB and the diverse CHD subtypes, Table S5: List of datasets used for epigenetic characterization of significant loci, Table S6: Annotation of epigenetic features in selected SNPs, Table S7: eQTL mapping performed in colocalizing susceptibility loci.

Author Contributions: Conceptualization, M.C., A.I., G.L., M.G.R.; methodology, A.T., V.A.L, M.D., M.C.; software, A.T., V.A.L.; formal analysis, A.T.; investigation, A.T., M.C.; resources, S.D., J.M., B.K., H.J.C.; data curation, S.D., J.M., B.K., H.J.C.; writing-original draft preparation, A.T., M.D., M.C.; writing-review and editing, A.T., M.D., H.J.C.; visualization, F.C., S.C., A.C., M.A.; supervision, M.D., M.C.; project administration, M.C.; funding acquisition, M.C., A.I., F.C., J.M., B.K.

Funding: This study was supported by grants from Associazione Italiana per la Ricerca sul Cancro (Grant no. 19255 to M.C. and Grant 20757 to A.I.); Ministero della Salute (GR-2011-02348722 to M.C.), Fondazione Italiana per la Lotta al Neuroblastoma (to M.C.); Associazione Oncologia Pediatrica e Neuroblastoma (to M.C.) and Fondazione Umberto Veronesi (to F.C.); NIH HHS/United States: R01 CA124709/CA/NCI, R01 CA180692 and R35 CA220500 (to J.M.); B.K. is supported by a British Heart Foundation Personal Chair.

Conflicts of Interest: The authors declare no conflict of interest. The funders had no role in the design of the study; in the collection, analyses, or interpretation of data; in the writing of the manuscript, or in the decision to publish the results.

\section{References}

1. Maris, J.M. Recent advances in neuroblastoma. N. Engl. J. Med. 2010, 362, 2202-2211. [CrossRef] [PubMed]

2. Capasso, M.; Diskin, S.J. Genetics and genomics of neuroblastoma. Cancer Treat. Res. 2010, 155, 65-84. [PubMed]

3. Tolbert, V.P.; Coggins, G.E.; Maris, J.M. Genetic susceptibility to neuroblastoma. Curr. Opin. Genet. Dev. 2017, 42, 81-90. [CrossRef] [PubMed]

4. Capasso, M.; Diskin, S.J.; Totaro, F.; Longo, L.; De Mariano, M.; Russo, R.; Cimmino, F.; Hakonarson, H.; Tonini, G.P.; Devoto, M.; et al. Replication of GWAS-identified neuroblastoma risk loci strengthens the role of BARD1 and affirms the cumulative effect of genetic variations on disease susceptibility. Carcinogenesis 2013, 34, 605-611. [CrossRef] [PubMed]

5. Capasso, M.; Diskin, S.; Cimmino, F.; Acierno, G.; Totaro, F.; Petrosino, G.; Pezone, L.; Diamond, M.; McDaniel, L.; Hakonarson, H.; et al. Common genetic variants in NEFL influence gene expression and neuroblastoma risk. Cancer Res. 2014, 74, 6913-6924. [CrossRef] [PubMed]

6. Capasso, M.; McDaniel, L.D.; Cimmino, F.; Cirino, A.; Formicola, D.; Russell, M.R.; Raman, P.; Cole, K.A.; Diskin, S.J. The functional variant rs34330 of CDKN1B is associated with risk of neuroblastoma. J. Cell Mol. Med. 2017, 21, 3224-3230. [CrossRef]

7. Cimmino, F.; Avitabile, M.; Diskin, S.J.; Vaksman, Z.; Pignataro, P.; Formicola, D.; Cardinale, A.; Testori, A.; Koster, J.; de Torres, C.; et al. Fine mapping of 2q35 high-risk neuroblastoma locus reveals independent functional risk variants and suggests full-length BARD1 as tumor-suppressor. Int. J. Cancer 2018, 143, 2828-2837. [CrossRef]

8. Tennant, P.W.; Pearce, M.S.; Bythell, M.; Rankin, J. 20-year survival of children born with congenital anomalies: a population-based study. Lancet 2010, 375, 649-656. [CrossRef]

9. Cordell, H.J.; Bentham, J.; Topf, A.; Zelenika, D.; Heath, S.; Mamasoula, C.; Cosgrove, C.; Blue, G.; Granados-Riveron, J.; Setchfield, K.; et al. Genome-wide association study of multiple congenital heart disease phenotypes identifies a susceptibility locus for atrial septal defect at chromosome 4p16. Nat. Genet. 2013, 45, 822-824. [CrossRef]

10. LaHaye, S.; Corsmeier, D.; Basu, M.; Bowman, J.L.; Fitzgerald-Butt, S.; Zender, G.; Bosse, K.; McBride, K.L.; White, P.; Garg, V. Utilization of Whole Exome Sequencing to Identify Causative Mutations in Familial Congenital Heart Disease. Circ. Cardiovasc. Genet. 2016, 9, 320-329. [CrossRef]

11. Jin, S.C.; Homsy, J.; Zaidi, S.; Lu, Q.; Morton, S.; DePalma, S.R.; Zeng, X.; Qi, H.; Chang, W.; Sierant, M.C.; et al. Contribution of rare inherited and de novo variants in 2,871 congenital heart disease probands. Nat. Genet. 2017, 49, 1593-1601. [CrossRef] [PubMed] 
12. Zaidi, S.; Brueckner, M. Genetics and Genomics of Congenital Heart Disease. Circ. Res. 2017, 120, $923-940$. [CrossRef] [PubMed]

13. Bellah, R.; D'Andrea, A.; Darillis, E.; Fellows, K.E. The association of congenital neuroblastoma and congenital heart disease. Is there a common embryologic basis? Pediatr. Radiol. 1989, 19, 119-121. [CrossRef] [PubMed]

14. Friedman, D.M.; Dunnigan, A.; Magid, M.S. Coarctation of the aorta associated with neuroblastoma. Pediatr. Cardiol. 1998, 19, 480-481. [CrossRef] [PubMed]

15. McElhinney, D.B.; Reddy, V.M.; Feuerstein, B.G.; Marx, G.R.; Hanley, F.L. Intraoperative discovery of neuroblastoma in an infant with pulmonary atresia. Ann. Thorac. Surg. 1997, 64, 1827-1829. [CrossRef]

16. Rosti, L.; Lin, A.E.; Frigiola, A. Neuroblastoma and congenital cardiovascular malformations. Pediatrics 1996, 97, 258-261. [PubMed]

17. Faingold, R.; Babyn, P.S.; Yoo, S.J.; Dipchand, A.I.; Weitzman, S. Neuroblastoma with atypical metastases to cardiac and skeletal muscles: MRI features. Pediatr. Radiol. 2003, 33, 584-586. [CrossRef] [PubMed]

18. George, R.E.; Lipshultz, S.E.; Lipsitz, S.R.; Colan, S.D.; Diller, L. Association between congenital cardiovascular malformations and neuroblastoma. J. Pediatr. 2004, 144, 444-448. [CrossRef]

19. Van Engelen, K.; Merks, J.H.; Lam, J.; Kremer, L.C.; Backes, M.; Baars, M.J.; van der Pal, H.J.; Postma, A.V.; Versteeg, R.; Caron, H.N.; et al. Prevalence of congenital heart defects in neuroblastoma patients: A cohort study and systematic review of literature. Eur. J. Pediatr. 2009, 168, 1081-1090. [CrossRef]

20. Kratz, C.P.; Rapisuwon, S.; Reed, H.; Hasle, H.; Rosenberg, P.S. Cancer in Noonan, Costello, cardiofaciocutaneous and LEOPARD syndromes. Am. J. Med. Genet. C Semin. Med. Genet. 2011, 157C, 83-89. [CrossRef]

21. Keyte, A.; Hutson, M.R. The neural crest in cardiac congenital anomalies. Differentiation 2012, 84, 25-40. [CrossRef] [PubMed]

22. Lombardo, R.C.; Porollo, A.; Cnota, J.F.; Hopkin, R.J. Congenital heart disease and aortic arch variants associated with mutation in PHOX2B. Genet. Med. 2018, 20, 1538-1543. [CrossRef] [PubMed]

23. Mosse, Y.P.; Laudenslager, M.; Khazi, D.; Carlisle, A.J.; Winter, C.L.; Rappaport, E.; Maris, J.M. Germline PHOX2B mutation in hereditary neuroblastoma. Am. J. Hum. Genet. 2004, 75, 727-730. [CrossRef] [PubMed]

24. Jiang, M.; Stanke, J.; Lahti, J.M. The connections between neural crest development and neuroblastoma. Curr. Top. Dev. Biol. 2011, 94, 77-127. [PubMed]

25. McDaniel, L.D.; Conkrite, K.L.; Chang, X.; Capasso, M.; Vaksman, Z.; Oldridge, D.A.; Zachariou, A.; Horn, M.; Diamond, M.; Hou, C.; et al. Common variants upstream of MLF1 at 3q25 and within CPZ at 4p16 associated with neuroblastoma. PLoS Genet. 2017, 13. [CrossRef] [PubMed]

26. Delaneau, O.; Howie, B.; Cox, A.J.; Zagury, J.F.; Marchini, J. Haplotype estimation using sequencing reads. Am. J. Hum. Genet. 2013, 93, 687-696. [CrossRef] [PubMed]

27. Howie, B.; Fuchsberger, C.; Stephens, M.; Marchini, J.; Abecasis, G.R. Fast and accurate genotype imputation in genome-wide association studies through pre-phasing. Nat. Genet. 2012, 44, 955-959. [CrossRef] [PubMed]

28. Das, S.; Forer, L.; Schönherr, S.; Sidore, C.; Locke, A.E.; Kwong, A.; Vrieze, S.I.; Chew, E.Y.; Levy, S.; McGue, M.; et al. Next-generation genotype imputation service and methods. Nat. Genet. 2016, 48, 1284-1287. [CrossRef] [PubMed]

29. Marchini, J.; Howie, B.; Myers, S.; McVean, G.; Donnelly, P. A new multipoint method for genome-wide association studies by imputation of genotypes. Nat. Genet. 2007, 39, 906-913. [CrossRef] [PubMed]

30. Chang, C.C.; Chow, C.C.; Tellier, L.C.; Vattikuti, S.; Purcell, S.M.; Lee, J.J. Second-generation PLINK: Rising to the challenge of larger and richer datasets. Gigascience 2015, 4, 7. [CrossRef] [PubMed]

31. Elliott, K.S.; Chapman, K.; Day-Williams, A.; Panoutsopoulou, K.; Southam, L.; Lindgren, C.M.; Arden, N.; Aslam, N.; Birrell, F.; Carluke, I.; et al. Evaluation of the genetic overlap between osteoarthritis with body mass index and height using genome-wide association scan data. Ann. Rheum. Dis. 2013, 72, 935-941. [CrossRef] [PubMed]

32. Duggal, P.; Gillanders, E.M.; Holmes, T.N.; Bailey-Wilson, J.E. Establishing an adjusted $p$-value threshold to control the family-wide type 1 error in genome wide association studies. BMC Genom. 2008, 9, 516. [CrossRef] [PubMed]

33. Pickrell, J.K.; Berisa, T.; Liu, J.Z.; Ségurel, L.; Tung, J.Y.; Hinds, D.A. Detection and interpretation of shared genetic influences on 42 human traits. Nat. Genet. 2016, 48, 709-717. [CrossRef] [PubMed]

34. Ahmed, M.; Sallari, R.C.; Guo, H.; Moore, J.H.; He, H.H.; Lupien, M. Variant Set Enrichment: An R package to identify disease-associated functional genomic regions. BioData Min. 2017, 10, 9. [CrossRef] [PubMed] 
35. Ward, L.D.; Kellis, M. HaploReg: A resource for exploring chromatin states, conservation, and regulatory motif alterations within sets of genetically linked variants. Nucleic Acids Res. 2012, 40, D930-D934. [CrossRef] [PubMed]

36. LinDA. Available online: http://linda.irgb.cnr.it/index.html (accessed on 26 April 2019).

37. Winsvold, B.S.; Nelson, C.P.; Malik, R.; Gormley, P.; Anttila, V.; Vander Heiden, J.; Elliott, K.S.; Jacobsen, L.M.; Palta, P.; Amin, N.; et al. Genetic analysis for a shared biological basis between migraine and coronary artery disease. Neurol. Genet. 2015, 1, e10. [CrossRef] [PubMed]

38. Fehringer, G.; Kraft, P.; Pharoah, P.D.; Eeles, R.A.; Chatterjee, N.; Schumacher, F.R.; Schildkraut, J.M.; Lindström, S.; Brennan, P.; Bickeböller, H.; et al. Cross-Cancer Genome-Wide Analysis of Lung, Ovary, Breast, Prostate, and Colorectal Cancer Reveals Novel Pleiotropic Associations. Cancer Res. 2016, 76, 5103-5114. [CrossRef] [PubMed]

39. Solovieff, N.; Cotsapas, C.; Lee, P.H.; Purcell, S.M.; Smoller, J.W. Pleiotropy in complex traits: Challenges and strategies. Nat. Rev. Genet. 2013, 14, 483-495. [CrossRef]

40. Kar, S.P.; Beesley, J.; Amin Al Olama, A.; Michailidou, K.; Tyrer, J.; Kote-Jarai, Z.; Lawrenson, K.; Lindstrom, S.; Ramus, S.J.; Thompson, D.J.; et al. Genome-Wide Meta-Analyses of Breast, Ovarian, and Prostate Cancer Association Studies Identify Multiple New Susceptibility Loci Shared by at Least Two Cancer Types. Cancer Discov. 2016, 6, 1052-1067. [CrossRef]

41. Hackinger, S.; Trajanoska, K.; Styrkarsdottir, U.; Zengini, E.; Steinberg, J.; Ritchie, G.R.S.; Hatzikotoulas, K.; Gilly, A.; Evangelou, E.; Kemp, J.P.; et al. Evaluation of shared genetic aetiology between osteoarthritis and bone mineral density dentifies SMAD3 as a novel osteoarthritis risk locus. Hum. Mol. Genet. 2017, 26, 3850-3858. [CrossRef]

42. Claussnitzer, M.; Dankel, S.N.; Kim, K.H.; Quon, G.; Meuleman, W.; Haugen, C.; Glunk, V.; Sousa, I.S.; Beaudry, J.L.; Puviindran, V.; et al. FTO Obesity Variant Circuitry and Adipocyte Browning in Humans. N. Engl. J. Med. 2015, 373, 895-907. [CrossRef] [PubMed]

43. Li, Y.R.; Li, J.; Zhao, S.D.; Bradfield, J.P.; Mentch, F.D.; Maggadottir, S.M.; Hou, C.; Abrams, D.J.; Chang, D.; Gao, F.; et al. Meta-analysis of shared genetic architecture across ten pediatric autoimmune diseases. Nat. Med. 2015, 21, 1018-1027. [CrossRef] [PubMed]

44. Luan, M.; Shang, Z.; Teng, Y.; Chen, X.; Zhang, M.; Lv, H.; Zhang, R. The shared and specific mechanism of four autoimmune diseases. Oncotarget 2017, 8, 108355-108374. [CrossRef] [PubMed]

45. Lupo, P.J.; Schraw, J.M.; Desrosiers, T.A.; Nembhard, W.N.; Langlois, P.H.; Canfield, M.A.; Copeland, G.; Meyer, R.E.; Brown, A.L.; Chambers, T.M.; et al. Association Between Birth Defects and Cancer Risk Among Children and Adolescents in a Population-Based Assessment of 10 Million Live Births. JAMA Oncol. 2019, in press. [CrossRef] [PubMed]

46. Tribulo, C.; Aybar, M.J.; Nguyen, V.H.; Mullins, M.C.; Mayor, R. Regulation of Msx genes by a Bmp gradient is essential for neural crest specification. Development 2003, 130, 6441-6452. [CrossRef] [PubMed]

47. Chen, Y.H.; Ishii, M.; Sun, J.; Sucov, H.M.; Maxson, R.E., Jr. Msx1 and Msx2 regulate survival of secondary heart field precursors and post-migratory proliferation of cardiac neural crest in the outflow tract. Dev. Biol. 2007, 308, 421-437. [CrossRef]

48. Revet, I.; Huizenga, G.; Chan, A.; Koster, J.; Volckmann, R.; van Sluis, P.; Øra, I.; Versteeg, R.; Geerts, D. The MSX1 homeobox transcription factor is a downstream target of PHOX2B and activates the Delta-Notch pathway in neuroblastoma. Exp. Cell Res. 2008, 314, 707-719. [CrossRef]

49. Ozcan, A.; Acer, H.; Ciraci, S.; Gumus, H.; Karakukcu, M.; Patiroglu, T.; Ozdemir, M.A.; Unal, E. Neuroblastoma in a Child with Wolf-Hirschhorn Syndrome. J. Pediatr. Hematol. Oncol. 2017, 39, e224-e226. [CrossRef]

50. Hoffmann, S.; Schmitteckert, S.; Griesbeck, A.; Preiss, H.; Sumer, S.; Rolletschek, A.; Granzow, M.; Eckstein, V.; Niesler, B.; Rappold, G.A. Comparative expression analysis of Shox2-deficient embryonic stem cell-derived sinoatrial node-like cells. Stem Cell Res. 2017, 21, 51-57. [CrossRef]

51. Hoffmann, S.; Clauss, S.; Berger, I.M.; Weiß, B.; Montalbano, A.; Röth, R.; Bucher, M.; Klier, I.; Wakili, R.; Seitz, H.; et al. Coding and non-coding variants in the SHOX2 gene in patients with early-onset atrial fibrillation. Basic Res. Cardiol. 2016, 111, 36. [CrossRef]

52. Blaschke, R.J.; Monaghan, A.P.; Schiller, S.; Schechinger, B.; Rao, E.; Padilla-Nash, H.; Ried, T.; Rappold, G.A. SHOT, a SHOX-related homeobox gene, is implicated in craniofacial, brain, heart, and limb development. Proc. Natl. Acad. Sci. USA 1998, 95, 2406-2411. [CrossRef] [PubMed] 
53. Vickerman, L.; Neufeld, S.; Cobb, J. Shox2 function couples neural, muscular and skeletal development in the proximal forelimb. Dev. Biol. 2011, 350, 323-336. [CrossRef] [PubMed]

54. Li, X.; Liu, H.; Gu, S.; Liu, C.; Sun, C.; Zheng, Y.; Chen, Y. Replacing Shox2 with human SHOX leads to congenital disc degeneration of the temporomandibular joint in mice. Cell Tissue Res. 2014, 355, 345-354. [CrossRef] [PubMed]

55. Rangrez, A.Y.; Pott, J.; Kluge, A.; Frauen, R.; Stiebeling, K.; Hoppe, P.; Sossalla, S.; Frey, N.; Frank, D. Myeloid leukemia factor-1 is a novel modulator of neonatal rat cardiomyocyte proliferation. Biochim. Biophys. Acta Mol. Cell Res. 2017, 1864, 634-644. [CrossRef] [PubMed]

56. Capasso, M.; Devoto, M.; Hou, C.; Asgharzadeh, S.; Glessner, J.T.; Attiyeh, E.F.; Mosse, Y.P.; Kim, C.; Diskin, S.J.; Cole, K.A.; et al. Common variations in BARD1 influence susceptibility to high-risk neuroblastoma. Nat. Genet. 2009, 41, 718-723. [CrossRef] [PubMed]

57. Cimmino, F.; Formicola, D.; Capasso, M. Dualistic Role of BARD1 in Cancer. Genes 2017, 8, 375. [CrossRef] [PubMed]

(C) 2019 by the authors. Licensee MDPI, Basel, Switzerland. This article is an open access article distributed under the terms and conditions of the Creative Commons Attribution (CC BY) license (http://creativecommons.org/licenses/by/4.0/). 